\title{
List of several SDN switches that support Group Table ALL feature
}

\author{
Mohammad Noormohammadpour and Cauligi S. Raghavendra \\ Ming Hsieh Department of Electrical Engineering, University of Southern California
}

\begin{abstract}
We list some Software Defined Networking (SDN) products that support the Group Table ALL feature which can be used to forward incoming packets to multiple outgoing ports via packet replication.
\end{abstract}

\section{SDN SWITCHES}

When using Group Tables, each group entry can have multiple action buckets each programmed with a set of actions. The "ALL" feature means all action buckets of a group entry will be executed and each bucket will be supplied with a copy of incoming packet that matches the group entry predicates. This feature has been in OpenFlow standard since version 1.1 and was added for the purpose of flooding, broadcasting or multicasting [1].

Although in the standard, this feature has not been widely supported by switch vendors. Recently, physical switches on the market have started providing support for this feature. We merely list several products that currently support this feature and cite related documents which contain detailed information on how these features are actually supported (e.g., maximum number of entries, maximum action buckets per entry and whether group chaining is allowed).

Group Tables ALL can be used for building multicast forwarding trees. Earlier multicasting approaches focus on connectivity as an objective [2]. Using SDN, one can build more efficient trees according to network topology and conditions as well as data properties. While assigning trees to data flows, in addition to reducing bandwidth usage, several objectives include reducing completion times [3], [4], minimizing deadline miss rate [5], [6], and balancing link utilization [7].

\section{REFERENCES}

[1] B. Pfaff et al., "OpenFlow Switch Specification Version 1.1.0 Implemented (Wire Protocol 0x02)," 2011. [Online]. Available: http://archive.openflow.org/documents/openflow-spec-v1.1.0.pdf

[2] S. Deering, "Host Extensions for IP Multicasting," Internet Requests for Comments, pp. 1-16, 1989. [Online]. Available: https://tools.ietf.org/html//rfc1112

[3] J. Cao, C. Guo, G. Lu, Y. Xiong, Y. Zheng, Y. Zhang, Y. Zhu, C. Chen, and Y. Tian, "Datacast: A scalable and efficient reliable group data delivery service for data centers," IEEE Journal on Selected Areas in Communications, vol. 31, no. 12, pp. 2632-2645, 2013.

[4] M. Noormohammadpour, C. S. Raghavendra, S. Rao, and S. Kandula, "Dccast: Efficient point to multipoint transfers across datacenters," USENIX Workshop on Hot Topics in Cloud Computing (HotCloud), 2017.
Table I

SDN PRODUCTS WITH SUPPORT FOR OFPGT_ALL

\begin{tabular}{|c|c|}
\hline Vendor & Product \\
\hline HP [8] & HP 5920 \& 5900 Switch Series \\
\hline HP [9] & HP 5130 EI Switch Series \\
\hline HP [10] & $\begin{array}{l}\text { HP Switch } 2920 \text { series, HP Switch } \\
3500 \text { series, HP Switch } 3800 \text { series, HP } \\
\text { Switch } 5400 \text { series, v1 and v2 modules, } \\
\text { HP Switch 5406R series, HP Switch } \\
5412 \text { A series, HP Switch } 6200 \text { series, } \\
\text { HP Switch } 6600 \text { series, HP Switch } \\
8200 \text { series, v1 and v2 modules }\end{array}$ \\
\hline $\begin{array}{ll}\text { Juniper } & \text { Networks } \\
{[11]} & \end{array}$ & $\begin{array}{l}\text { MX Series, EX9200, QFX5100 and } \\
\text { EX4600 }\end{array}$ \\
\hline Alcatel-Lucent [12] & $\begin{array}{l}\text { OmniSwitch 10K, OmniSwitch 9900, } \\
\text { OmniSwitch 6900, OmniSwitch 6860, } \\
\text { and OmniSwitch } 6865\end{array}$ \\
\hline IBM [13] & $\begin{array}{l}\text { IBM System Networking RackSwitch } \\
\text { G8264 }\end{array}$ \\
\hline Brocade [14] & $\begin{array}{l}\text { Brocade VDX 2741, Brocade VDX } \\
6740 \text {, Brocade VDX } 6940 \text { and Brocade } \\
\text { VDX } 8770\end{array}$ \\
\hline
\end{tabular}

[5] M. Noormohammadpour and C. S. Raghavendra, "DDCCast: Meeting Point to Multipoint Transfer Deadlines Across Datacenters using ALAP Scheduling Policy," Department of Computer Science, University of Southern California, Tech. Rep. 17-972, 2017.

[6] S. Ji, "Resource optimization across geographically distributed datacenters," Master's thesis, University of Toronto, 2017.

[7] A. Iyer, P. Kumar, and V. Mann, "Avalanche: Data center multicast using software defined networking," International Conference on Communication Systems \& Networks (COMSNETS), pp. 1-8, 2014.

[8] HP 5920 \& 5900 Switch Series OpenFlow Command Reference. [Online]. Available: http://h20565.www2.hpe.com/hpsc/doc/public/display?sp4ts. oid $=5221896 \&$ docId $=$ emr $\% 5$ Fna- c04089449\&docLocale $=$ en $\% 5 F U S$

[9] HP 5130 EI Switch Series OpenFlow Configuration Guide. [Online]. Available: http://h20565.www2.hpe.com/hpsc/doc/ public/display?sp4ts.oid $=7399420 \&$ docLocale $=$ en $\% 5 F U S \&$ docId $=$ emr\%5Fna-c04771714

[10] HP OpenFlow 1.3 Administrator Guide Wired Switches K/KA/KB/WB 15.15. [Online]. Available: http://h20564.www2.hpe. $\mathrm{com} / \mathrm{hpsc} / \mathrm{doc} / \mathrm{public} /$ display?docId=c04217797\&lang=en-us\&cc=us

[11] OpenFlow v1.3.1 Compliance Matrix for Devices Running Junos OS. [Online]. Available: https://www.juniper.net/documentation/en\%5FUS/junos/topics/ reference/general/junos-sdn-openflow-v1.3.1-compliance-matrix. html\#table-openflow-compliance-matrix-group-types

[12] OmniSwitch AOS Release 8 Switch Management Guide. [Online]. Available: http://enterprise.alcatel-lucent.com/assets/ documents/omniswitch-8-switch-management-guide.pdf

[13] IBM System Networking RackSwitch G8264 Application Guide For Networking OS 7.9. [Online]. Available: http://www-01.ibm.com/ support/docview.wss?uid=isg3T7000679\&aid=1

[14] Network OS Software Defined Networking (SDN) Configuration Guide Supporting Network OS 7.0.0. [Online]. Available: http://www.brocade.com/content/dam/common/ documents/content-types/configuration-guide/nos-700-sdnguide.pdf 